\title{
Editorial
}

\section{A Look Back at NRMP 2020 and the Road Ahead}

Frederick M Schiavone, MD, FACEP', 2

\section{Abstract}

Author affiliations are listed at the end of this article.

Correspondence to:

Frederick Schiavone, MD

Division Vice President, GME and DIO USF Morsani College of Medicine GME Programs HCA Healthcare West Florida Division, 3031 N Rocky Point Drive W, \#400 Tampa, FL 33607 (Frederick.Schiavone@ hcahealthcare.com)

\section{Keywords}

internship and residency; education, medical, graduate; personnel selection; achievement; behavior and behavior mechanisms

ahead to the Supplemental Offer and Acceptance Program (SOAP)-allowing unfilled programs and unmatched applicants to find each other in 3 rounds of SOAP, before Friday's traditional Match Day.

As we comb through Match data-which we all do-we examine which specialties are growing in number, which are growing or fading in popularity and which are seeing changing demographics. In the first ("most desirable") category, the specialties that filled $100 \%$ of available positions in the Match were Dermatology, Medicine-Emergency Medicine, Neurological Surgery, Physical Medicine \& Rehabilitation (C), Integrated Plastic Surgery, and Thoracic Surgery. Another measure of the attractiveness of the specialties includes a high percentage (> $80 \%$ ) of available positions filled by US seniors. This year, Integrated Plastic Surgery, Medicine-Pediatrics, Neurological Surgery, Orthopedic Surgery, Otolaryngology, Thoracic Surgery and Vascular Surgery increased in popularity among US seniors. The NRMP also reported Family Medicine, Internal Medicine, Pathology, Primary Care Pediatrics, and Preliminary Surgery were less popular with US seniors. With the national data as a backdrop, we look introspectively at our programs results in the specialties called out by the NRMP

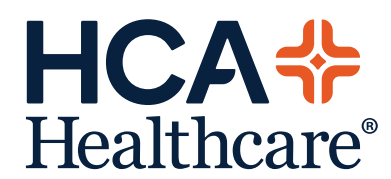

www.hcahealthcarejournal.com

(c) 2020 HCA Physician Services, Inc. d/b/a Emerald Medical Education
HCA Healthcare Journal of Medicine 
as growing or fading in popularity, or those attracting more or fewer US seniors. ${ }^{1}$

As we look ahead to next year's recruitment cycle, we know a wholesale change is coming on the horizon. In February 2020, the Association of American Medical Colleges announced that the USMLE program would "change score reporting for Step 1 from a three-digit numeric score to reporting only a pass/fail outcome" and that a "numeric score will continue to be reported for Step 2 Clinical Knowledge (CK) and Step 3. Step 2 Clinical Skills (CS) will continue to be reported as Pass/Fail." They announced at this time that the policy would take effect no later than January 1, 2022. ${ }^{2}$ It is not an overstatement to say that this changes everything. As published in 2011 in the Journal of Surgical Education, George Makdisi et al. concluded the following when writing, How we select our residents-a survey of selection criteria in general surgery residents. "Even though all general surgery programs have a wide range of screening/selection criteria, USLME Step 1 is the single most important factor for preliminary screening." The authors go on to state, "the interview is the most important factor in determining the final selection. The final selection is relatively subjective and based on a combination of interview, USLME scores, research experience, and personal judgment."

So, acknowledging that the USMLE Step 1 three-digit numeric score is the single most important factor in determining who to invite to interview with our programs, where does this leave us for recruitment of the entering class of 2022? With what information will we be able to screen the applicants if this metric is removed? As we review Medical Student Performance Evaluations with standard narratives, descriptions of clerkship performance that are generic and medical school transcripts that frequently only contain pass/fail grades, how are we to determine unique characteristics that differentiate candidates from each other? Without increasing the number of interview invitations we extend to compensate for the lack of the USMLE Step 1 three-digit numeric scores, how do we utilize the information we do have to include or exclude candidates to consider?
As we approach the 20 months ahead, we need to consider how we can work with the medical school community, so that we can include more behavioral characteristics that allow us to identify candidates that are a good fit for our residency programs. I propose that we need to have more data from the medical schools than they are currently providing, qualitative and quantitative data, and scores that allow us to predict residency success. How do we gather evidence that relates to what we genuinely need to entrust our patients with resident physicians who prioritize patient safety and quality, teaming ability, professionalism, altruism and integrity?

We need to construct a rubric that will help align the evaluation of success in medical school with a prediction of success in our individual residency training programs. We need enough evidence to ensure the students we select for interviews and finally for ranking can demonstrate some success in the competencies and milestones against which their progress in GME will be measured. We look forward to identifying what information can be extracted from medical school performance in order to support our "best fit" conclusions.

\section{Conflicts of Interest}

The author declares he has no conflicts of interest.

Dr. Frederick Schiavone is an employee of HCA Healthcare West Florida Divison, an organization affiliated with the journal's publisher.

This research was supported (in whole or in part) by HCA Healthcare and/or an HCA Healthcare affiliated entity. The views expressed in this publication represent those of the author(s) and do not necessarily represent the official views of HCA Healthcare or any of its affiliated entities.

\section{Author Affiliations}

1. USF Morsani College of Medicine GME Programs

2. HCA Healthcare West Florida Division 


\section{References}

1. National Resident Matching Program. Press

Release: Thousands of Medical Students

and Graduates Celebrate NRMP Match

Results. Accessed April 22, 2020. http:// www.nrmp.org/2020-press-release-thousands-resident-physician-applicants-celebrate-nrmp-match-results/

2. United States Medical Licensing Examination. Change to pass/fail score reporting for Step 1. Accessed April 22, 2020. https://www.usmle.org/ inCus/

3. Makdisi G, Takeuchi T, Rodriguez J, Rucinski J, Wise L. How We Select Our Residents-A Survey of Selection Criteria in General Surgery Residents. J Surg Edu. 2011;68:67-72. https://doi. org/10.1016/j.jsurg.2010.10.003 